Communication: Lipases were used to restore partially the strength losses of cotton fabrics crosslinked with 1,2,3,4-butanetetracarboxylic acid. The enzymatic hydrolysis of the ester linkages at low temperature and neutral $\mathrm{pH}$ resulted in $10 \%$ strength recovery, coupled with a slight deterioration of the crease-resistance effect. The conventional alkaline hydrolysis provided higher strength recovery, however provoked considerable change in the durable press performance of the fabrics.

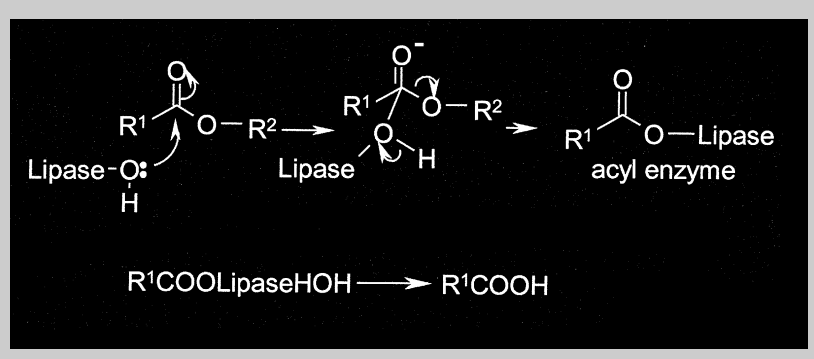

Mechanism of the lipase catalysis.

\title{
Lipases to Improve the Performance of Formaldehyde- Free Durable Press Finished Cotton Fabrics
}

\author{
Tzanko Tzanov, ${ }^{1}$ Maria Stamenova, ${ }^{2}$ Rossitza Betcheva, ${ }^{2}$ Artur Cavaco-Paulo $* 1$ \\ ${ }^{1}$ Departamento de Engenharia Têxtil, Universidade do Minho, Campus de Azurém, 4800-058 Guimarães, Portugal \\ Fax: +351 253 510293; E-mail: artur@det.uminho.pt \\ ${ }^{2}$ University of Chemical Technology and Metallurgy, 1756 Sofia, Bulgaria
}

Keywords: butanetetracarboxylic acid; crosslinking; lipases; strength recovery

\section{Introduction}

Nowadays the fabrics produced from cotton fibers have the largest share in the textile market. However, their crease-resistance is not satisfactory to meet the contemporary requirements for "easy-care" textile materials. When cotton fabric is deformed, the numerous $\mathrm{H}$-bonds which exist between cellulose molecules are destroyed and consecutively created fixing the new deformed state of the fabric. The conventional durable press finishes to improve the crease-resistance of cotton fabrics are mostly achieved on the basis of a condensation reaction at high temperature between $N$-methylol compounds, such as dimethyloldihydroxyethylene urea (DMDHEU) or its derivatives and cellulose. However, the formaldehyde, which is released during the fixation procedure, has been identified as a potential human carcinogen. In the last years many efforts have been made to develop formaldehyde-free crosslinking agents. For this purpose some product with lower formaldehyde content have been suggested, i.e., $\alpha$-hydroxyalkylamides, polymers from urea and glutaraldehyde, and diamidodihydroxyethane products, all of them crosslinking the cellulose trough ether bond formation. ${ }^{[1,2]}$ The most promising formaldehyde free reagents, however, are the polycarboxylic acids 1,2,3,4-cyclopentanetetracarboxylic acid, 1,2,3,4-butane- tetracarboxylic acid (BTCA), or a combination of citric acid and polymers of maleic acid. The reaction with the cellulose substrate proceeds trough ester crosslinking, catalyzed by sodium hypophosphite. ${ }^{[3,4]}$ A major drawback of any crease-resistance finish on cotton is the decreased fabric strength, which increases as the degree of crosslinking increases. The crosslinking of cellulose reduces the mobility of the macromolecules and greater stress, which could not be uniformly distributed along the polymer chains, is accumulated in the fabric structure, leading to its disruption. The crosslinking of cellulose molecules with polycarboxylic acids causes partially reversible strength loss and some irreversible acid catalyzed degradation of cellulose at elevated temperature. ${ }^{[5-7-16]}$ In other words, the strength loss of cotton esterified with polycarboxylic acid is a consequence of both acid depolymerisation and crosslinking. While the fabric strength loss caused by degradation is an irreversible process, the ester crosslinks might be removed by alkaline hydrolysis, gradually restoring the strength loss. The alkaline treatment could cause an undesirable reduction in the durable press performance of the finish fabrics. A milder, ecologically clean, and controlled hydrolysis of the ester linkages could be achieved using enzymes. Lipases (triacyl glycerol acyl hydrolases EC 3.1.1.3) are 


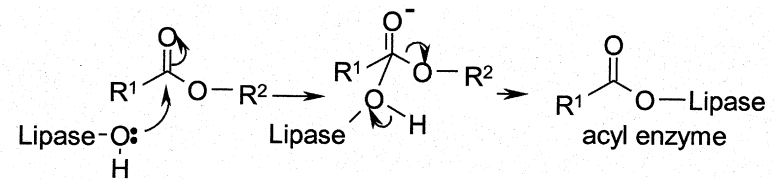

$\mathrm{R}^{1} \mathrm{COOLipaseHOH} \longrightarrow \mathrm{R}^{1} \mathrm{COOH}$

Scheme 1. Mechanism of the lipase catalysis.

an unique class of hydrolases, ${ }^{[17,18]}$ which are catalytically active in water, in mixtures of water and a water-immiscible organic solvent and in organic solvent, catalyzing both hydrolysis and esterification reactions. The large excess of water favors the hydrolysis reaction. Most of lipases are serine hydrolases, containing a serine residue in their active site, and forming an intermediate acylenzyme substrate complex (Scheme 1).

The objective of this research was to provide an efficient biocatalytic ester hydrolysis to restore partially the strength loss of polycarboxylic durable press finished cotton fabrics, without deteriorating the crease-resistance effect.

\section{Experimental Part}

\section{Textile Material and Durable Press Finish}

The fabric used in this study was a desized, scoured and bleached cotton. The cotton fabric was impregnated on foulard (100\% wet pick-up) with a solution containing 1,2,3,4butanetetracarboxylic acid (BTCA) (from Aldrich), and anhydrous sodium hypophosphite (from Sigma) as the catalyst, with a BTCA/catalyst ratio of $3: 2$ wt.-\%. ${ }^{[8,13]}$ The impregnated fabric was dried at $80^{\circ} \mathrm{C}$ for $5 \mathrm{~min}$ and then cured in a Werner Mathis curing machine at $180^{\circ} \mathrm{C}$ for $2 \mathrm{~min}$. Afterward the treated fabric was washed extensively to remove the remaining unreacted acid and catalyst.

\section{Enzyme Treatment}

Lipase (EC 3.1.1.3) type VII from Candida rugosa, 724 units $\cdot \mathrm{mg}^{-1}$ solid, was purchased from Sigma and applied to the crosslinked cotton fabrics in different concentrations and for different time at $\mathrm{pH} 7.5$ (0.1 M phosphate buffer) and $37^{\circ} \mathrm{C}$, as indicated by the producer, in a shaker with $100 \mathrm{rpm}$.

\section{Wrinkle Recovery Angle, Tensile Strength, FT-IR Analysis and Dyeability with Cationic Dyes}

Wrinkle recovery angle (WRA) and tensile strength of the BTCA treated fabrics were measured according to AATCC test method 66-1990 and ASTM method D5035-90, respectively. Diffuse reflectance spectra of some of the samples were collected by Bomem MB-series FT-IR spectrometer, performing 100 scans for each spectrum. No smoothing function and baseline correction were applied. Potassium bromide was used to obtain the background spectrum. The effectiveness of the lipase deesterification was assessed com- paring the ester carbonyl band intensity at $1724 \mathrm{~cm}^{-1} \cdot{ }^{[8-15]}$ The fabrics were treated for $2 \mathrm{~min}$ at room temperature with $0.1 \mathrm{M} \mathrm{NaOH}$ to convert the free carboxyl to carboxylate. Otherwise the carboxyl carbonyl band overlaps the ester carbonyl band. ${ }^{[11]}$ The lipase treated samples $(1 \mathrm{~g}$ each) were dyed with $20 \cdot 10^{-6}$ M C.I. Basic Dye 9 purchased from Farmitalia Carlo Erba S.p.A., at $50^{\circ} \mathrm{C}$, for $1 \mathrm{~h}$, in bath to fabric ratio 50:1. The color of the dyed fabrics was evaluated using a reflectance measuring Datacolor apparatus at standard illuminant $\mathrm{D}_{65}$ (LAV/Spec. Incl., d/8, $\left.\mathrm{D}_{65} / 10^{\circ}\right)$ in terms of $K / S$ values. The Kubelka-Munk relationship $(K / S)$, where $K$ is an adsorption coefficient and $S$ is a scattering coefficient, is applied to textiles under the assumption that light scattering is due to the fibers, while adsorption of light is due to the colorant.

\section{Results and Discussion}

\section{Enzyme Concentration}

The effect of the enzyme concentration on tensile strength and wrinkle recovery angle (WRA) of BTCA treated fabrics was investigated. Tensile strength normally increases as the hydrolysis of the crosslinked fabric progresses. In our experiment the tensile strength of the BTCA crosslinked fabrics increased when up to $0.4 \mathrm{~g} \cdot \mathrm{1}^{-1}$ lipase was applied for hydrolysis of the ester bonds. Above this enzyme concentration a further improvement of the fabric tensile performance was not observed (Figure 1). This limitation of the hydrolysis could be explained with the restricted accessibility to the enzymatic attack of the BTCA/cellulose ester crosslinks in the dense cellulose structure. The ester groups have a slightly higher concentration in the interior of the fabric than in its near surface. ${ }^{[9]}$ The large enzyme molecule is not able to get in contact with the substrate and to form the intermediate enzyme-substrate complex. The dimension of the protein molecule is a self-limiting factor for undesirable further hydrolysis of the BTCA/cellulose crosslinkages and lessening of the crease-resistance effect.

It is known that the electrostatic enzyme/substrate interactions play an important role in the enzyme catalysis. It was found that the active site of Candida rugosa lipases becomes negatively charged at $\mathrm{pH}^{[19]}$ (in our experiments the lipases were applied at $\mathrm{pH}$ 7.5). The negative charge in the active site is needed for the expulsion of the negatively charged hydrolysis products and is therefore essential for the maximum activity of the enzyme. After cleavage of the ester bond, an ionized acid will be ejected from the active site due to electrostatic repulsion. If the active site was positively charged, a product inhibition would occur due to interaction of the active site with the negatively charged polycarboxylic acid. In the case of crosslinking of cellulose with polycarboxylic acids, four ester bonds per acid molecule are possible. Even though the enzyme hydrolyzed some of these ester crosslinkages, the product still remains on the fabric 


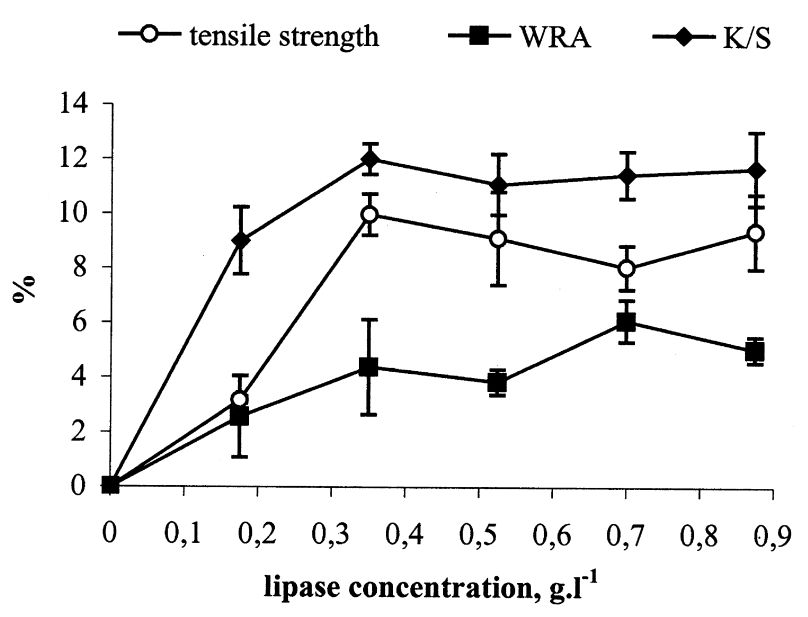

Figure 1. Tensile strength recovery, WRA decrease and decrease in dyeabilitywith cationic dye of BTCA treated cotton (6\% BTCA/4\% $\mathrm{NaH}_{2} \mathrm{PO}_{2}$ ), hydrolyzed by different concentration of lipase for $1 \mathrm{~h}$.

due to singly bonded acid side groups. ${ }^{[9]}$ Thus hydrolysis of the ester groups is faster than the rate of BTCA molecule removal. With the progress of the hydrolysis reaction the fabric will become more negatively charged and the enzyme will be rejected from the fabric's surface, impeding further hydrolysis. As more hydrolysis occurs, the intermediate binding between enzyme and substrate becomes more difficult due to electrostatic repulsion. This might be another explanation, apart from the steric constraints, why the hydrolysis did reach a maximum and afterwards remained steady independently on the enzyme concentration.

A comparatively mild chemical hydrolysis was performed using $0.1 \mathrm{M} \mathrm{NaOH}$, at $37^{\circ} \mathrm{C}$ for $1 \mathrm{~h}$. The alkaline hydrolysis was accompanied with considerable loss of the desired durable press effect - nearly $40 \%$ decrease of WRA vs. $30 \%$ of strength improvement. At the same conditions, samples treated with enzyme showed about $4 \%$ decrease of wrinkle recovery angle (WRA), which corresponds to nearly $10 \%$ strength recovery (Figure 1 ). Even though BTCA molecules were gradually removed from the fabric as a result of hydrolysis, the effectiveness of the remaining BTCA molecules as a crosslinking agent decreased only slightly. The change of WRA was twice lower than the tensile strength recovery of BTCA crosslinked cotton.

The dyeing with basic dye (methylene basic blue 9) is an indirect indication for the state of the enzymatic process. The dyeability of BTCA treated fabrics decreased after $1 \mathrm{~h}$ hydrolysis with up to $0.4 \mathrm{~g} \cdot \mathrm{l}^{-1}$ lipase (Figure 1). Significant correlation could be found between the tensile strength recovery and the decrease in dyeability of the hydrolyzed cotton. We expected that the presence offree carboxyl and carboxylate groups on the BTCA treated cotton after the cleavage of the ester bonds would make it dyeable with basic dyes. However, the removal of the

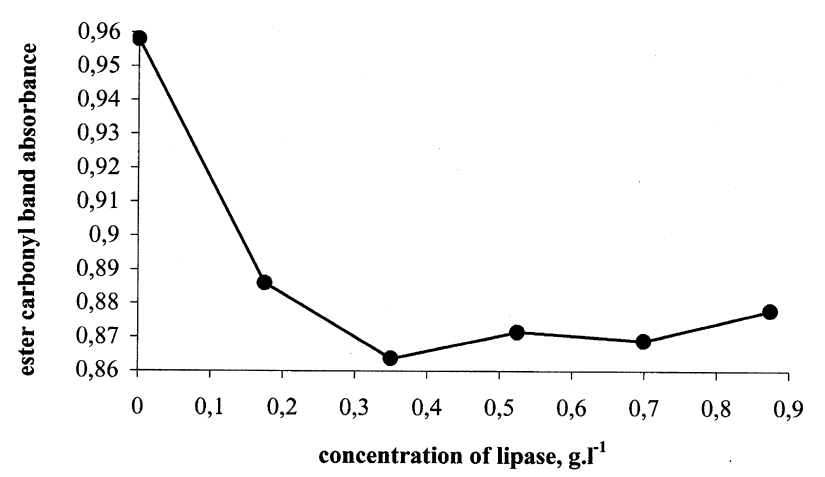

Figure 2. Ester carbonyl band absorbance at $1724 \mathrm{~cm}^{-1}$ of cotton treated with $6 \%$ BTCA/4\% $\mathrm{NaH}_{2} \mathrm{PO}_{2}$ and hydrolyzed with different concentrations of lipase.

product during the enzymatic hydrolysis reduces the affinity of the cationic dye toward the fabric. The alkali hydrolyzed BTCA cotton was dyed the worst (about 50\% decrease of $K / S$ ), while the untreated cotton was not dyeable with cationic dyes.

The IR spectroscopy data in Figure 2 show a decrease of the intensity of the ester carbonyl peak at $1724 \mathrm{~cm}^{-1}$ with increasing lipase concentration. Above $0.4 \mathrm{~g} \cdot \mathrm{l}^{-1}$ lipase, where the highest tensile strength recovery was observed, the curve of the carbonyl band intensity reached a plateau, e.g. no further hydrolysis occurred.

\section{Hydrolysis Time}

At fixed concentration $\left(0.4 \mathrm{~g} \cdot \mathrm{1}^{-1}\right)$ the effect of the hydrolysis time on fabric tensile performance and crease-resistance was also examined. The process time was varied from $30 \mathrm{~min}$ to $25 \mathrm{~h}$. The tensile strength recovery reached a maximum during the first hour of the process and remained constant for the next $24 \mathrm{~h}$ of treatment (Figure 3). The decrease of WRA follows the same tendency. Inactivation of the enzyme by the hydrolysis products and long operational time is equally possible, apart from

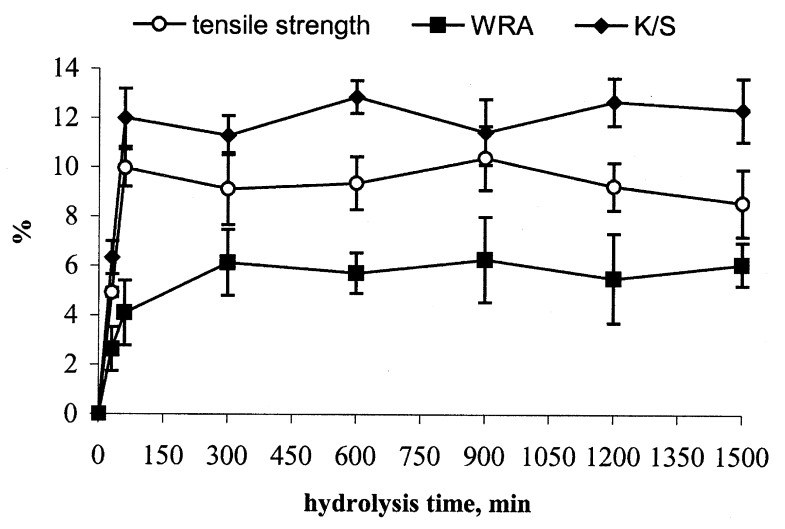

Figure 3. Tensile strength recovery, WRA decrease and decrease in dyeability with cationic dye of BTCA treated fabrics $\left(6 \% \mathrm{BTCA} / 4 \% \mathrm{NaH}_{2} \mathrm{PO}_{2}\right)$, hydrolyzed by $0.4 \mathrm{~g} \cdot 1^{-1}$ lipase for different times. 


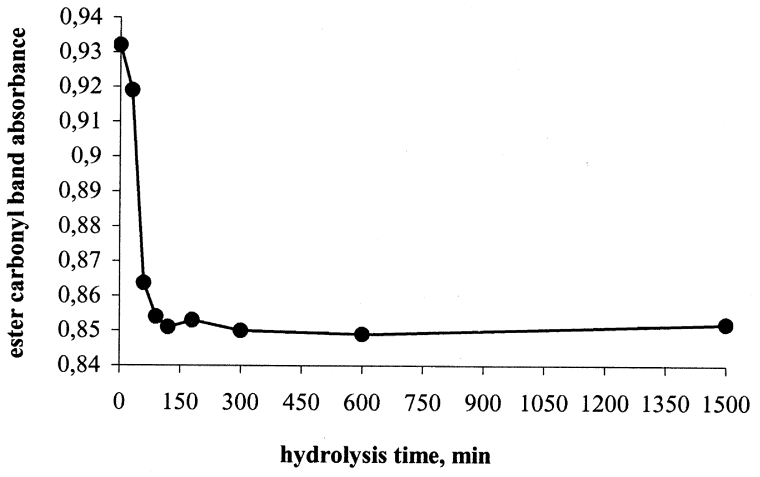

Figure 4. Ester carbonyl band absorbance at $1724 \mathrm{~cm}^{-1}$ of cotton, treated with $6 \% \mathrm{BTCA} / 4 \% \mathrm{NaH}_{2} \mathrm{PO}_{2}$ and hydrolyzed with $0.4 \mathrm{~g} \cdot \mathrm{l}^{-1}$ lipase for different times.

the above stated steric and electrostatic considerations. The decrease of the intensity of the ester carbonyl peak in the IR spectrum of the fabric corresponds to the inhibition of the enzyme hydrolysis (Figure 4).

\section{Conclusion}

In this research a safe, ecologically acceptable enzymatic hydrolysis process to restore the tensile strength of BTCA treated cotton fabrics was established, as an alternative to the conventional alkaline treatment. The application of lipases in low concentration for short treatment time restored partially the strength loss of the fabrics due to the polycarboxylic crosslinking. The lipase treatment improved the tensile strength with up to $10 \%$, causing just $4 \%$ alteration of the crease-resistance of the fabrics. A steady state of the tensile recovery was reached, possibly due to steric difficulties to form the enzyme/substrate intermediate complex, or due to electrostatic repulsion between the negatively charged binding/catalytic site of the lipase and the increasing negative charge of the cotton during the hydrolysis. The alkaline hydrolysis of the BTCA durable press finish resulted in substantial reduction of the crease-resistance effect, whose deterioration overcomes the gain in tensile strength. Data from FT-IR spectroscopy confirmed the occurrence and then the inhibition of the hydrolysis of the polycarboxylic crosslinkages. With proceeding enzymatic hydrolysis the dyeability of the BTCA treated cotton decreased due to the removal of the product.

Acknowledgement: We would like to thank the HPMF-CT2000-00868 project for providing the grant to Dr. Tzanov.

Received: February 14, 2002

Revised: April 22, 2002

Accepted: May 29, 2002

[1] J. Frick, R. Harper, Text. Res. Inst. 1982, 2, 141

[2] D. Lämmermann, Melliand Textilber. 1992, 73, 274.

[3] US Patent 4,820,307 (1989), United States of America, Department of Agriculture, invs.: C. Welch, B. Andrews.

[4] C. Welch, Text. Res. J. 1988, 8, 480.

[5] I.-S. Kang, C. Q. Yang, W. Wei, G. Lickfield, Text. Res. J. 1998, 68, 865

[6] W. Wei, C. Q. Yang, Y. Jiang, Text. Chem. Color. 1999, 31,34 .

[7] W. Wei, C. Q. Yang, Text. Res. J. 1999, 69, 145.

[8] C. Q. Yang, G. Bakshi, Text. Res. J. 1996, 66, 377.

[9] C. Q. Yang, Text. Res. J. 1991, 61, 298.

[10] C. Q. Yang, Text. Res. J. 2001, 71, 201.

[11] C. Q. Yang, Text. Res. J. 1991, 61, 433.

[12] C. Q. Yang, Z. Mao, G. Lickfield, Text. Chem. Color. 2000, 32, 43 .

[13] C. Q. Yang, X. Wang, Text. Res. J. 1996, 66, 595.

[14] C. Q. Yang, X. Wang, I.-S. Kang, Text. Res. J. 1997, 67, 334.

[15] C. Q. Yang, L. Xu, S. Li, Y. Jiang, Text. Res. J. 1998, 68, 457.

[16] C. Q. Yang, D. Wang, Text. Res. J. 2000, 70, 615.

[17] "Lipases: Structure, Mechanism and Genetic Engineering”, L. Alberghina, R. D. Schmid, R. Verger, Eds., VCH, Weinheim 1990.

[18] B. Borgström, H. L. Brockman, "Lipases", Elsevier, Amsterdam 1984.

[19] M. Petersen, P. Fojan, S. Petersen, J. Biotechnol. 2001, 85, 115. 


\section{Graphical Abstract for the Contents Page: Please check}

Communication: Lipases are used to partially restore the strength of cotton fabrics crosslinked with 1,2,3,4-butanetetracarboxylic acid. Enzymatic hydrolysis of the ester linkages at low temperature and neutral $\mathrm{pH}$ results in a $10 \%$ strength recovery, associated with slight deterioration of the creaseresistance effect. Conventional alkaline hydrolysis provides higher strength recovery, but causes considerable changes in the durable press performance of the fabrics. The mechanism of the lipase-catalyzed ester hydrolysis is outlined in the Scheme.

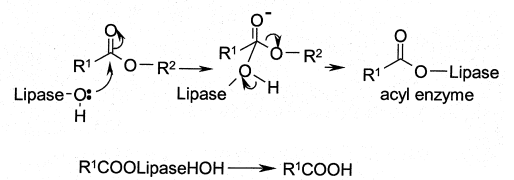

Lipases to Improve the Performance of Formaldehyde-Free Durable Press Finished Cotton Fabrics

T. Tzanov, M. Stamenova, R. Betcheva, A. Cavaco-Paulo*

Macromol. Mater. Eng. 2002, 287, 462-465 